\title{
Psychotropic drug use in patients with various chronic somatic diseases
}

\author{
Lia Boelman, MD \\ Hugo M Smeets, PhD \\ Mirjam J Knol, PhD \\ Arjan W Braam, PhD, MD \\ Mirjam I Geerlings, PhD \\ Niek J de Wit, PhD, MD \\ University Medical Centre Utrecht, \\ Julius Center for Health Sciences and \\ Primary Care, Utrecht \\ THE NETHERLANDS
}

\begin{abstract}
Background and Objectives: To reveal insight into prevalence and incidence of psychotropic drug use in patients with various chronic somatic diseases and into time differences between incidence of the somatic disease and start of psychotropic drug use.

Methods: A cohort study was performed using the Agis Health Database from 2002 to 2005. Patients with one or more of nine frequently occurring chronic somatic diseases were selected $(\mathrm{N}=140,983)$. The non-exposed group $(\mathrm{N}=417,486)$ was composed of patients without any of these nine diseases. For the somatic disease prescribed drugs were used as a proxy-indicator. Prevalences and incidences of psychotropic drug use were calculated as well as mean time between the incidence of the somatic disease and release date of the psychotropic drug.

Results: Prevalence of antidepressant drugs and benzodiazepines was increased among patients with somatic diseases, with odds ratios of respectively 1.83 (95\% CI 1.80-1.87) and 2.31 (95\% CI 2.27-2.35). In the first year after incidence of the somatic disease, mean duration in time to start of psychotropic drug use was significantly shorter compared to patients without somatic disease.

Conclusions: Physicians should be aware of the high prevalence and earlier onset of psychotropic drug use in all patients with chronic somatic diseases.
\end{abstract}

Received: 13 May 2011

Revised: 3 May 2012

Accepted: 31 May 2012

Acronyms used in text: $\mathrm{ATC}=$ anatomical therapeutic chemical; $\mathrm{CI}=$ confidence interval; $\mathrm{DDD}=$ defined daily dose; GP = general practitioner; NSAID = non-steroidal anti-inflammatory drug; OR = odds ratio. 


\section{Introduction}

Mental disorders are a significant general health problem. In the Netherlands as many as four in ten adults suffer from a mental disorder once in their life ${ }^{1,2}$. Although the majority $(86 \%)$ of the patients are treated in primary care by their general practitioner (GP), many patients with a mental disorder are unrecognized by their $\mathrm{GP}^{1,3-6}$. Mental disorders are known to be co-morbid with a wide range of chronic somatic diseases, including musculoskeletal, cardiovascular, pulmonary diseases and diabetes ${ }^{7-9}$. This comorbidity has a negative impact on quality of life, subjective suffering, mortality and health costs ${ }^{10-13}$. Appropriate treatment may improve both course and outcome of the mental disorder and the co-morbid somatic disease as well as quality of life ${ }^{14,15}$.

In Europe as well as the United States the prevalence of psychotropic drug use increased in the last 20 years $^{16,17}$. Uncertain is how somatic comorbidity in mental disorders influence GPs pharmacological treatment decisions. Some studies suggest that comorbidity of somatic and mental disorders increases the likelihood of prescribing drugs for mental disorders ${ }^{6,18}$. In contrast, others found that the presence of a somatic disease has a negative effect on prescribing of psychotropic drugs ${ }^{19}$.

Up to now little is known about the temporal relation between somatic diseases and psychotropic drug use. There may be differences in duration in time till onset of the psychotropic drug use between various chronic somatic diseases. Possible explanations for these differences could be on the one hand failure of adaptation strategies on the short term or on the other hand the impact of the disease on the long term.
The current study was designed to reveal insight into prevalence and incidence of psychotropic drug use in patients with various chronic somatic diseases and whether an incident somatic disease might lead to earlier start of psychotropic drug use. Our main research questions were:

1. What is the prevalence and incidence of psychotropic drug use in patients with various chronic somatic diseases?

2. What is the mean duration in time between the incidence of the chronic somatic disease and the start of psychotropic drug use?

\section{Methods}

\section{Database}

We selected our subjects from the Health Database of Agis, a major health insurance company in the Netherlands and the database contains demographic, socio-economic and health consumption data. The Agis Health Database represents an accurate registration of drug prescriptions, independent of prescriber, since all prescriptions must be registered in this database before costs can be reimbursed. In the Netherlands, almost all drugs used in this study are only available as prescription drugs. Only acetaminophen, NSAIDs and antacid are also available overthe-counter. We used data from January $1^{\text {st }}$, 2002 to December 31 $1^{\text {st }}, 2005$.

\section{Chronic somatic diseases}

Nine chronic somatic diseases were studied (box 1). Prescribed drugs were used as a proxy-indicator for the presence of these diseases, using the Anatomical Therapeutic Chemical (ATC) classification system code of the prescribed drugs. Patients were divid- 
ed into groups with one, two or $\geq$ three chronic somatic diseases. Patients with one chronic somatic disease were further divided into nine groups (pulmonary disease, diabetes mellitus, cardio-vascular disease, rheumatoid arthritis, cancer, dyspeptic disorders, inflammatory bowel disease, musculoskeletal disorders and urological disorders).

Box 1

ATC codes used for the various diseases an psychotropic drugs

Chronic somatic disease

Pulmonary disease

Diabetes mellitus

Cardio-vascular disease

Rheumatoid arthritis

Cancer

Dyspeptic disease

Inflammatory bowel disease

Musculoskeletal disease

Urological disease ATC codes $\mathrm{R} 03 \mathrm{~A}^{*}, \mathrm{R} 03 \mathrm{~B} *, \mathrm{R} 03 \mathrm{D} *$

A10A*, A10B*

$\mathrm{C} 01 \mathrm{~A}^{*}, \mathrm{C} 1 \mathrm{~B}^{*}, \mathrm{C} 01 \mathrm{D} *, \mathrm{C}^{*} *, \mathrm{C} 07^{*}, \mathrm{C} 08^{*}, \mathrm{C} 09^{*}$

A07EC01, L01BA01, L01XC02, L04AA01, L04AA11, L04AA12, L04AA13, L04AA14, L04AA17, L04AX01, L04AX03, M01CB*, P01BA01, P01BA02

$\mathrm{LO2}^{*}, \mathrm{~L} 01 *^{*}$

$\mathrm{A} 02 \mathrm{~A} *, \mathrm{~A} 02 \mathrm{~B} *$

A07EA*, A07EC*, L04AA01, L04AA12, L04AX01, L04AX03, L01BA01

M01A*, N02AA59, N02AX*, N02BE*

G04B*, G04C*

\begin{tabular}{ll}
\hline Psychotropic drug use & ATC codes \\
\hline Benzodiazepines & N05BA*, N05CD*, N05CF* \\
Antidepressant drugs & N06A* \\
Antipsychotic drugs & N05A* \\
\hline
\end{tabular}

\section{Patients}

We selected all patients, regardless there age, who were prescribed 180 Defined Daily Doses (DDDs) (assumed average dose per day for each drug) or more per year for the mentioned somatic diseases during at least three of the four years (January $1^{\text {st }}, 2002$ to December $\left.31^{\text {st }}, 2005\right)$. This strict criterion of three to four years of at least 180 DDDs per year was used to make sure that we included true chronically ill patients.

Patients who used from 1 to 179 DDDs for the mentioned somatic diseases or more than 180 DDDs per year but for a shorter period of time than three of the four years were excluded. All patients with none of the selected chronic somatic conditions in the years 2002 to 2005 were selected as the non-exposed group.

In the chronic somatic disease groups defined above, a sub-sample was selected of newly ill patients, who were prescribed the first drug for a chronic somatic disease in the year 2002 (no prescription of the drug in the year 2001), who used 180 DDDs or more per year during the three following years (2003 to 2005) for the same somatic disease and who didn't use any psychotropic drug in the year 2001. Date of first prescription for 
the somatic drug, was defined as start of the somatic disease. In case of comorbidity of somatic diseases the first incident somatic disease was used to clarify possible differences between the various chronic somatic diseases. Exclusion of patients using psychotropic drugs in 2001 was done to select only patients with new psychotropic drug use after incidence of the somatic disease. A random subsample of the non-exposed (twice the number), composed of patients not using any psychotropic drug in the year 2002, was selected as a reference for the sub-sample of new chronically ill patients.

\section{Psychotropic drug use}

Psychotropic drug use was defined as the use of any benzodiazepine, antidepressant drug of antipcyhotic drug in the years 2002 to 2005 (box 1).

\section{Covariables}

Age at January $1^{\text {st }}, 2002$, gender, ethnicity and socioeconomic status were used as covariables. Ethnicity is not registered in the Agis Health Database, but foreign nationality is. The first generation Moroccan and Turkish immigrants was selected by their nationality. To select second and third generation immigrants, the surnames of the selected first generation immigrants were matched with the remaining of the database and visually controlled on origin of the name. The social status of the living area and the insurance category were used as rough indicators for socioeconomic status. To define the social status of the living area we used the postal code, as defined and registered by the Statistics Netherland ${ }^{20}$. Patients were divided into four insurance categories (paid employment, old-age pension, work disabled and social insurance).

\section{Data-analysis}

Differences between chronic somatic disease groups and no chronic somatic disease group were calculated using chi-square tests (for covariables gender, ethnicity, social status of living area and insurance category) and two-tailed independent t-tests (for covariable age).

Comorbidity between the chronic somatic disease and psychotropic drug use was analyzed by calculating prevalences of total psychotropic drug use, as well as prevalences of subgroups of psychotropic drug use for the years 2002 to 2005. Logistic regression was used to calculate crude, and -after control for covariables- adjusted odds ratios of psychotropic drug use in the different somatic disease groups.

We calculated mean duration in time (in days) between first prescriptions of the somatic drug to the first prescription of any psychotropic drug. In the reference group without chronic somatic disease, mean duration in time (in days) from January, $1^{\text {st }} 2003$ to the first prescription of any psychotropic drug was calculated. First year follow up was from 0 to 365 days, second year follow up from 366 to 730 days and third year follow up from 731 to 1095 days. Because non-exposed have no specific onset of time in the year until the start of psychotropic drugs, one could expect they will start equally distributed over the year and mean duration in days will be close to 183 days (half of first year follow up), 584 (half of second year follow up) or 913 days (half of third year follow up). Incident psychotropic drug use was calculated when the first psychotropic drugs were prescribed in that year of the follow up. A linear regression model was used to analyze differences in time of the incidence of psychotropic drug use in relation to the various chronic somatic diseases. 
In the logistic and linear regression analysis the group with patients without chronic somatic disease was used as reference category. The analyses were done using SPSS version 14.0.1.

\section{Results}

A total number of 140,983 subjects with one or more chronic somatic disease were included. Table 1 describes the baseline characteristics by somatic disease of the exposed and non-exposed $(\mathrm{N}=417,486)$ group. Subjects in the non-exposed group were significantly younger compared with the exposed and were less often female (Table 1).

Rheumatoid arthritis and inflammatory bowel disease groups were relatively small, respectively 400 and 419 subjects, and cardio-vascular disease $(\mathrm{N}=70,625)$, dyspeptic disorders $(\mathrm{N}=21,027)$ and two chronic somatic disease $(\mathrm{N}=22,233)$ groups were relatively large. The most remarkable differences between the various chronic somatic diseases were: pulmonary disease, rheumatoid arthritis and inflammatory bowel dis-

Table 1

Characteristics of various chronic somatic disease groups and no chronic somatic disease group

\begin{tabular}{lccc} 
& Subjects $(\mathrm{N}=)$ & Age $($ mean $(\mathrm{SD}))$ & Female gender (\%) \\
\hline No chronic disease & 417486 & $40.6(15.9)$ & 52.4 \\
Chronic disease & 140983 & $62.2(14.2)^{*}$ & $60.6^{*}$ \\
- One chronic disease & 115830 & $61.9(14.4)^{*}$ & $60.9^{*}$ \\
Pulmonary disease & 9040 & $50.7(16.6)^{*}$ & $55.8^{*}$ \\
Cardiovascular disease & 70625 & $64.4(12.9)^{*}$ & $64.7^{*}$ \\
Diabetes mellitus & 6597 & $60.9(15.1)^{*}$ & 52.4 \\
Musculoskeletal disease & 3846 & $57.5(14.6)^{*}$ & $65.7^{*}$ \\
Urological disease & 2652 & $67.6(11.7)^{*}$ & $9.0^{*}$ \\
$\quad$ Rheumatoid arthritis & 419 & $49.4(15.5)^{*}$ & $67.3^{*}$ \\
Inflammatory bowel disease & 400 & $51.7(16.4)^{*}$ & $61.0^{*}$ \\
Dyspeptic disease & 21027 & $59.3(15.1)^{*}$ & $57.8^{*}$ \\
Cancer & 1224 & $64.3(14.7)^{*}$ & $74.3^{*}$ \\
- Two chronic diseases & 22233 & $65.7(12.4)^{*}$ & $60.0^{*}$ \\
- Three/more chronic diseases & 2920 & $66.2(11.5)^{*}$ & $55.4^{*}$ \\
\hline
\end{tabular}

$* \mathrm{P}<0.05$ compared with no chronic disease group.

ease patients were relatively younger compared with the other chronic somatic disease groups; urological disease patients were more often male and cancer patients more often female.

There was a higher risk of antidepressant, benzodiazepine and total psychotropic drug use in patients with chronic somatic diseases compared with patients without chronic somatic diseases, with adjusted odds ratios of respectively $1.83(95 \% \mathrm{CI} 1.80-1.87), 2.31$ (95\%CI 2.27-2.35) and 2.27 (95\%CI 2.242.31) (Table 2). The risk of antipsychotic drug use was slightly lower in patients with 


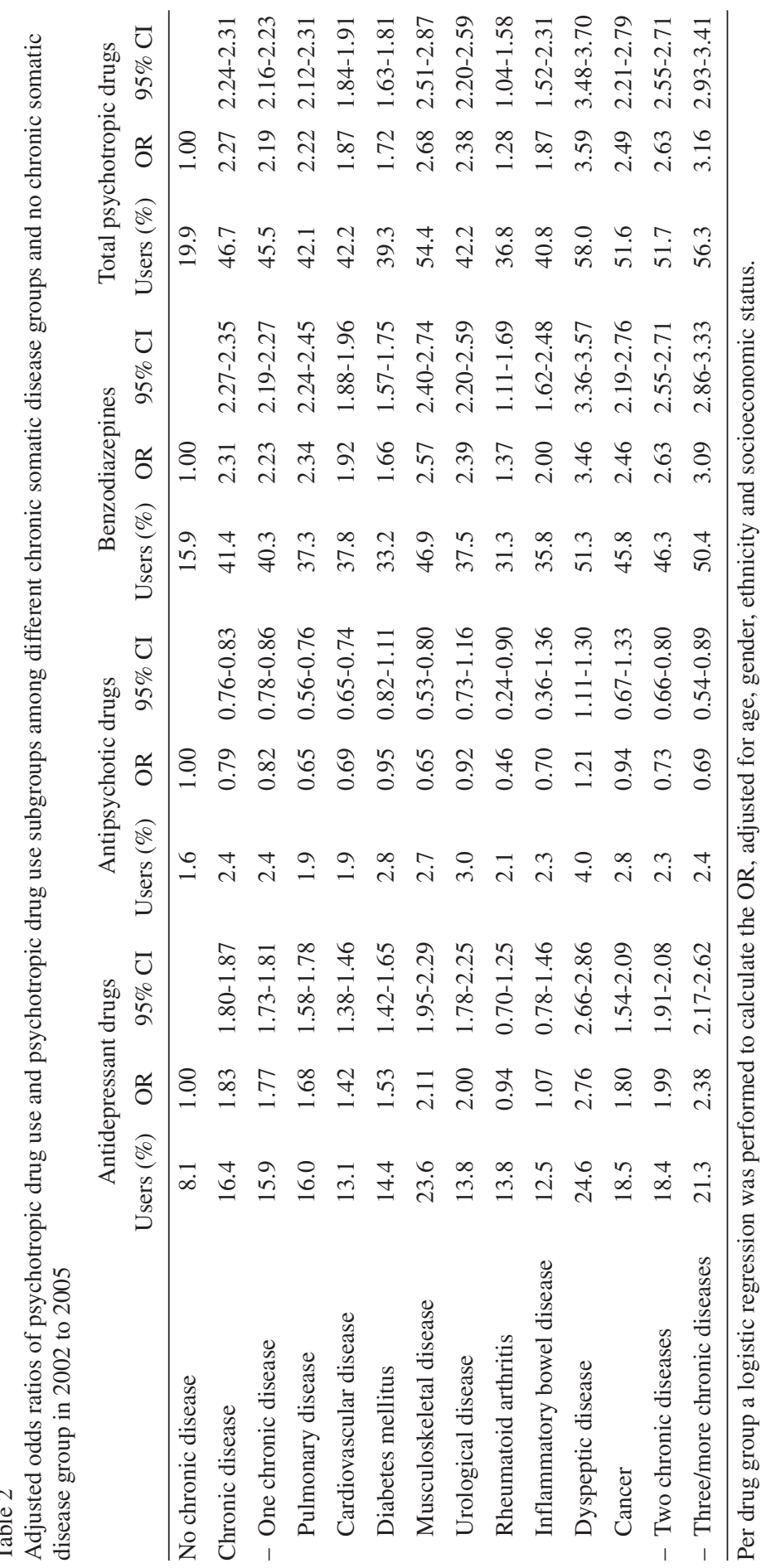


chronic somatic diseases compared with patients without chronic somatic diseases (adjusted odds ratio 0.79 (95\%CI 0.76-0.83)). The highest risk of psychotropic drug use compared with no chronic somatic disease group, was found in four groups: musculoskeletal disease (adjusted odds ratio 2.68 (95\%CI 2.51-2.87)), dyspeptic disease (adjusted odds ratio 3.59 (95\%CI 3.48-3.70)), two chronic somatic diseases (adjusted odds ratio $2.63(95 \%$ CI $2.55-2.71)$ ) and three or more chronic somatic diseases (adjusted odds ratio 3.16 (95\% CI 2.93-3.41)) (Table 2).

In 2002 a total number of 2,504 subjects developed a new chronic somatic disease (Table 3 ). Non-exposed patients were significantly younger and less often female (Table 3). Because of the small different somatic disease groups we show only the results of total patients with chronic somatic disease.

Table 3

Characteristics of subsample of new chronic somatic disease groups and no chronic somatic disease group

\begin{tabular}{llll} 
& Subjects $(\mathrm{N}=)$ & Age $($ mean $(\mathrm{SD}))$ & Female gender $(\%)$ \\
\hline No chronic disease & 5010 & $40.1(15.9)$ & 50.8 \\
Chronic disease & 2504 & $57.9(14.9)^{*}$ & $55.8^{*}$ \\
\hline
\end{tabular}

$* \mathrm{P}<0.05$ compared with no chronic disease group.

The three years incidence of psychotropic drug use in patients with a new chronic somatic disease was $18.8 \%$, compared with $10.0 \%$ in the non-exposed group (Figure 1).
Patients with new chronic disease developed more often a psychotropic drug use in the first year follow up compared with non-exposed (9.9\% to $4.1 \%$, Figure 1 and Table 4$)$. In the

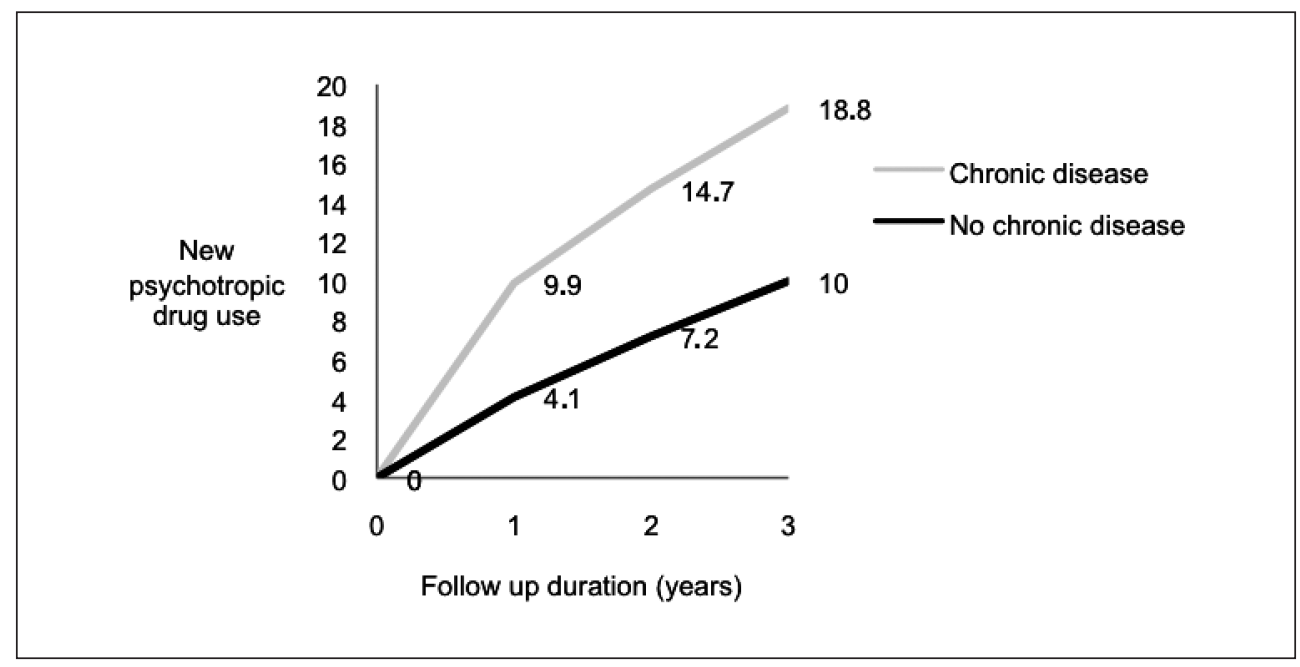

Figure 1. Trend of patients with new psychotropic drug use among patients with chronic somatic disease and patients without chronic somatic disease. 


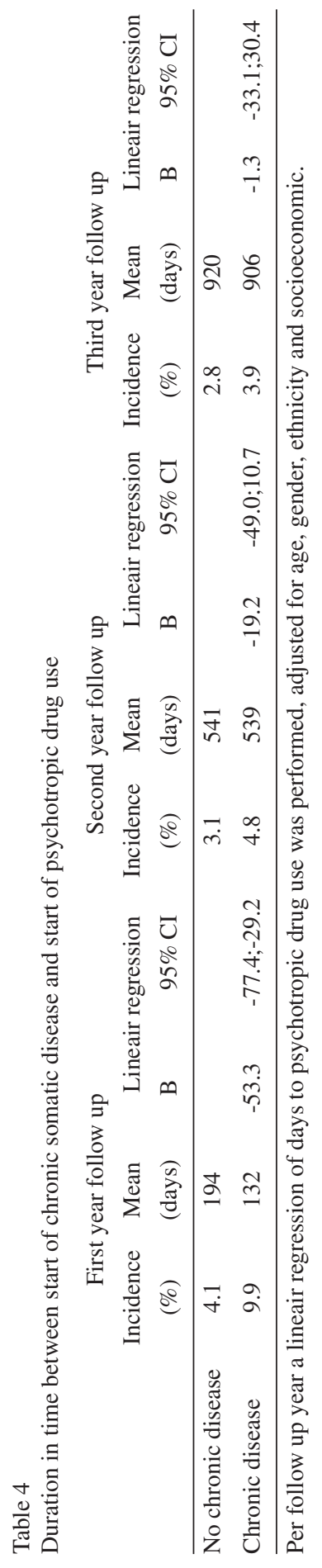

exposed, mean period in time from the incidence of the somatic disease to the incidence of psychotropic drug use during the first year follow up was 132 days (Table 4). This was significantly earlier compared with non-exposed (B is -53.3, 95\% CI -77.4 - -29.2). During the second and third year follow up there were no differences in mean period in time from the incidence of the somatic disease to start of psychotropic drug use.

\section{Discussion}

In this study we aimed to investigate the prevalence and incidence of psychotropic drug use in patients with various chronic somatic diseases and the duration in time till the onset of psychotropic drug use in these patients. Prevalence of antidepressant drug use was increased in all chronic somatic disease groups, except for rheumatoid arthritis and inflammatory bowel disease groups. Prevalence of benzodiazepine use was increased in all somatic disease groups. Incidence of psychotropic drug use was twice as high in patients with chronic somatic disease compared with non-exposed during the first year follow up. Mean duration in time from start of the somatic disease to start of psychotropic drug use was significantly shorter in the first year follow up in the exposed compared with non-exposed.

Our findings confirm the high prevalence of psychotropic drug use among patients with various chronic somatic diseases, but overall prevalences were slightly higher than those found in the general population ${ }^{7-9,21-23}$. A possible explanation for the higher prevalence rates is that we collected data of patients who were prescribed psychotropic drugs and did not diagnose patients with mental disease using standardized psychiatric instruments. In our study also patients with low dose psy- 
chotropic drugs, for example low dose benzodiazepines for a short term sleeping disorder, are taken into account in prevalence rates. This could have led to these slightly higher prevalences. On the other hand true prevalences of mental diseases could be even higher, because of the widespread under recognition of mental disorders ${ }^{1,4-6}$.

Unknown is if specific somatic diseases influence the likelihood of using psychotropic drugs in case of a mental disorder. Symptoms of somatic and mental disease might overlap, physicians and patients might believe that depression is a normal reaction to the somatic disease or physicians might be reluctant to prescribe psychotropic drugs because of potential side effects of poly-pharmacy or potential interactions and risks in certain somatic conditions (like the use of benzodiazepines in patients with severe pulmonary disease or tricyclic antidepressants in patients with cardiovascular disease) $)^{24}$. In one study, GPs were less likely to initiate any depression care in patients with pre-existing ischemic heart disease or cardiac arrhythmia, after a new diagnosis of depression, compared with patients without chronic disease $\mathrm{e}^{18}$. Most studies did not specifically investigate differences in psychotropic drug use in patients with various chronic somatic diseases. In our study patients with musculoskeletal, dyspeptic and more than one chronic somatic disease had a highest prevalence of psychotropic drug use. It might be that these higher prevalences can be partly explained by higher prescription rate of psychotropic drug in these particular groups of patients.

We showed that patients with somatic disease have an earlier onset of a psychotropic drug use during the first year follow up after the start of the somatic disease compared with patients without a chronic somatic disease. The short period between the start of the somatic disease and the start of a psychotro- pic drug use makes a physiological explanation or failure of coping strategies on the long term less likely. A possible explanation could be failing of coping and adaptation strategies on the short term among patients with somatic disease. Another explanation is that patients with a chronic somatic disease visit their physician more often, which increases the chance that the physician diagnoses the mental disorder and start psychotropic drug use.

An unexpected finding was that the patients with various chronic somatic diseases had a lower risk of antipsychotic drug prescription. This raises the question if psychotic disorders and treatment receive less attention in times of a chronic somatic conditions, especially pulmonary disease, cardiovascular disease and musculoskeletal symptoms. We think the lower prescription of antipsychotic drugs in these patients may be a reflection of a more carefully prescription policy of physicians in these patients, given the potential harmfully side effects of these psychotropic drugs in these patient groups or the interaction with medication for chronic disease. Nevertheless, under treatment among patients with schizophrenia represents a serious concern. This under treatment may be due to a lower quality of somatic healthcare in this particular group, but could also be caused by a lack of motivation among the patients due to the disorder (avolition) ${ }^{25,26}$.

Strengths of our study are the use of a large cohort with reference group and of day-today prescription data. We used prescription data to try to avoid the possible problems of underestimation and biased associations that might occur with the use of self reported questionnaires of somatic diseases that are used in earlier studies ${ }^{7-9,21,22,27,28}$. Limitations of this approach are that not all somatically ill patients are treated with medication and that there is no information available on the actual compliance with the medication. 
This may not be an exact approach of the real prevalence and could lead to some underestimation. Finally, we had to use prescription data as proxy indicators of somatic diseases as the AGIS database does not contain diagnostic information. Prescription data may not be an optimal reflection of the prevalence of somatic disease, as some drugs are used for different indications as well.

A second limitation is the overlap of medication use between some of the different somatic disease groups, for example use of methotrexate by patients with inflammatory bowel disease as well as patients with rheumatoid arthritis, and usage of acetaminophen and NSAIDs by patients with musculoskeletal disorders as well as with rheumatoid arthritis. This could have resulted in some misclassification of patients. We did not study dosages of psychotropic drug use because we focused on prevalence differences between different somatic disease groups. This method may have led to some overestimation of the prevalence of mental disorders.

Third, some of our prevalence rates, for example for diabetes (prevalence $40.1 \%$ in female and $41.6 \%$ in male in the Netherlands) and cardiovascular disease (prevalence $29.4 \%$ in female and $50.1 \%$ in male in the Netherlands) are relatively low ${ }^{29}$. This could be because many of these patients have comorbid disease and are classified in the groups with 2 or more chronic somatic diseases. This could have led to the relatively low ORs in diabetes and cardiovascular disease groups. Fourth, to select only chronically ill patients we used the criterion of 180 DDDs or more per year for the somatic diseases during at least three out of the four years. Because of this criterion it could be possible we selected mainly more severe chronically ill patients. Therefore, our results may be more applicable to the more severe chronically ill patients. Fifth, data are restricted to only nine frequently occurring groups of chronic somatic diseases. Patients in the exposed and the non-exposed groups might have had any comorbid somatic disease other than these nine. The exclusion of these more uncommon chronic somatic diseases may have caused an underestimation of the actual relation.

Finally we could not take the consultation rate into account as a possible confounder as data of physician consultation rate are not registered in the Agis Health Database. Most likely is that the consultation rate is lower among patients without a chronic somatic disease compared with patients with a chronic somatic disease. The higher consultation rate of chronically ill patients might have led to the earlier recognition a mental disorder and start of psychotropic drug use.

In conclusion, this study showed a higher prevalence antidepressant drug use and benzodiazepine use among patients with various chronic somatic diseases. General practitioners and psychiatrists should be aware of the high prevalence of psychotropic drug use in all patients with chronic somatic diseases, but especially in patients with musculoskeletal disorders, dyspeptic disorders and more than one chronic somatic disease. Possible explanations for the earlier start of a psychotropic drug use after a new somatic disease are earlier detection of a mental disorder because of higher consultation rate due to the new somatic disease or failing of coping or adaptation strategies on the short term.

\section{References}

1. Bijl RV, Ravelli A, van Zessen G. Prevalence of psychotropic disorders in the general population: results of The Netherlands Mental Health Survey an Incidence Study (NEMESIS). Soc Psychiatry Psychiatr Epidemiology 1998; 33: 587-595. 
2. De Graaf R, Ten Have M, Van Gool C, Van Dosselaer S. Prevalence of mental disorders, and trends from 1996 to 2009. Results from NEMESIS-2. Tijdschr Psychiatr 2012; 54: $27-38$.

3. Van Rijswijk E, Borghuis M, van de Lisdonk E, Zitman $\mathrm{F}$, van Weel C. Treatment of mental health problems in general practice: a survey of psychotropics prescribed and other treatments provided. Int J Clin Pharmacol Ther 2007; 45: 23-29.

4. Lecrubier Y. Widespread uderrecognition und undertreatment of anxiety and mood disorders: results from 3 European studies. J Clin Psychiatry 2007; 68 (Suppl 2): 3641.

5. Docherty JP. Barriers to the diagnosis of depression in primary care. J Clin Psychiatry 1997; 58 (Suppl 1): 5-10.

6. Nuyen J, Volkers AC, Verhaak PF, Schellevis FG, Groenewegen PP, Van den Bosch GA. Accuracy of diagnosing depression in primary care: the impact of chronic somatic and psychiatric co-moborbidity. Psychol Med 2005; 35: 11851195.

7. Harter M, Baumeister H, Reuter K, Jacobi F, Hofler M, Bengel J, et al. Increased 12-month prevalence rates of mental disorders in patients with chronic somatic diseases. Psychother Psychosom 2007; 76: 354-360.

8. Sareen J, Cox BJ, Clara I, Asmundson GJ. The relationship between anxiety disorders an physical disorders in the U.S. National Comorbidity Survey. Depress Anxiety 2005; 21: 193-202.

9. Scott KM, Bruffaerts R, Tsang A, Ormel J, Alonso J, Angermeyer MC, et al. Depression-anxiety relationships with chronic physical conditions: results from the World Mental Health Surveys. J Affect Disord 2007; 103: 113-120.

10. Cassano P, Fava M. Depression and public health: an overview. J Psychosom Res 2002; 53: 849-857.

11. Baumeister H, Balke K, Harter M. Psychiatric and somatic comorbidities are negatively associated with quality of life in physically ill patients. J Clin Epidemiol 2005; 58: 1090-1100.

12. Schmitz N, Wang J, Malla A, Lesage A. Joint effect of depression and chronic conditions on disability: results from a population-based study. Psychosom Med 2007; 69: 332-338.

13. Vinogradova Y, Coupland C, Hippisley-Cox C, Whyte $\mathrm{S}$, Penny C. Effects of severe mental illness on survival of people with diabetes. Br J Psychiatry 2010; 194: 272-277.

14. Stockton P, Gonzales JJ, Stern NP, Epstein SA. Treatment patterns and outcomes of depressed medically ill and non-medically ill patients in community psychiatric practice. Gen Hosp Psychiatry 2004; 26: 2-8.
15. Simon GE, Von Korff M, Lin E. Clinical and functional outcomes of depression treatment in patients with and without chronic medical illness. Psychol Med 2005; 35: 271-279.

16. Paulose-Ram R, Safran MA, Jonas BS, Gu Q, Orwig D. Trends in psychotropic medication use among U.S. adults. Pharmacoepidemiol Drug Saf 2007; 16: 560-570.

17. Carta MG, Kovess V, Hardoy MC, Brugha T, Fryers T, Lehtinen V, et al. Psychosocial wellbeing and pscychiatric care in the European Communities: analysis of macro indicators. Soc Psychiatry Pscyhiatr Epidemiol 2004; 39: 883-892.

18. Nuyen J, Spreeuwenberg PM, Van Dijk L, den Bos GA, Groenewegen PP, Schellevis FG. The influence of specific chronic somatic conditions on the care for co-morbid depression in general practive. Psychol Med 2008; 38: 265-277.

19. Tylee AT, Freeling P, Kerry S. Why do general practitioners recognize major depression in one woman patient yet miss it in another? Br J Gen Pract 1993; 43: 327-330.

20. Statistics Netherlands (CBS). Available at: www.cbs.nl

21. Verdurmen J, Ten Have M, Van Dorsselaer S, Van 't Land H, Vollebergh WAM, De Graaf R. Psychische Stoornissen bij Mensen met een Lichamelijke Aandoening. Resultaten van de 'Netherlands Mental Health Survey an Incidence Study’ (NEMESIS). Trimbos Institute; 2006.

22. Moussavi S, Chatterji S, Verdes E, Tandon A, Patel V, Ustun B. Depression, chronic diseases, and decrements in health: results from the World Health Surveys. Lancet 2007; 370: 851-858.

23. Bijl RV, Zesen G van, Ravelli A. Psychiatrische morbiditeit onder volwassenen in Nederland: het NEMESIS-onderzoek. II. Prevalentie van psychiatrische stoornissen. Ned Tijdsch Geneeskd 1997; 141: 2453-2460.

24. Bogner HR, Ford DE, Gallo JJ. The role of cardiovascular disease in the identification and management of depression by primary care physicians. Am J Geriatr Psychiatry $2006 ; 14: 71-78$.

25. Björkenstam E, Ljung R, Burström B, MittendorferRutz E, Hallqvist J, Weitaft GR. Quality of medical care and excess mortality in psychiatric patients-a nationwide registerbased study in Sweden. 2012 Feb 24; 2:e000778. Print 2012.

26. Laursen TM, Munk-Olsen T, Agerbo E, Gasse C, Mortensen PB. Somatic hospital contacts, invasive cardiac procedures, and mortality from heart disease in patients with severe mental disorder. Arch Gen Psychiatry 2009; 66: 713-720.

27. Goldman N, Lin IF, Weinstein M, Lin YH. Evaluating the quality of self-reports of hypertension and diabetes. J Clin Epidemiol 2003; 56: 148-154. 
28. Molenaar EA, Van Ameijden EJ, Grobbee DE, Numans ME. Comparison of routine care self-reported and biometrical data on hypertension and diabetes: results of the Utrecht Health Project. Eur J Public Health 2007; 17: 199-205.

29. Poos MJJC. Nationaal Kompas Volksgezondheid. RIVM. 2011

\author{
Corresponding author: \\ L. Boelman and H.M. Smeets \\ University Medical Centre Utrecht \\ Julius Center for Health Sciences and Primary Care \\ Str. 6.131 \\ PO Box 85500 \\ 3508 GA Utrecht \\ The Netherlands \\ Phone: 0031887553012 \\ Fax: 0031887555480 \\ E-mail: lboelman@gmail.com and \\ H.M.Smeets@umcutrecht.nl
}

